\title{
DEGENERAÇÃO HEPATOLENTICULAR: UM RELATO DE CASO
}

\section{HEPATOLENTICULAR DEGENERATION: A CASE REPORT}

\author{
Suzan Haruhi Muraoka ${ }^{1}$, Luciana Osório Cavalli ${ }^{2}$ \\ ${ }^{1}$ Acadêmica do Curso de Medicina do Centro Universitário Assis Gurgacz. ${ }^{2}$ Graduada em Medicina. \\ Mestrado pelo programa de Biociências e Saúde. \\ * Autor correspondente: shmuraoka@minha.fag.edu.br. ORCID: https://orcid.org/0000-0003-2523- \\ 4965.
}

\begin{abstract}
RESUMO
Introdução: a Degeneração Hepatolenticular, conhecida como Doença de Wilson (DW), trata-se de um distúrbio autossômico recessivo raro do metabolismo de cobre, com prevalência de 1:30.000 nascidos-vivos, acomete principalmente jovens adultos. Os pacientes podem apresentar distúrbios psicomotores, oftalmológicos, doenças psiquiátricas atípicas, hemólise inexplicada, doença hepática sem outra causa aparente. Relato e discussão do caso: Paciente de 27 anos, masculino, diagnosticado com DW aos 22 anos de idade. Iniciou o quadro com queixa de elevação de pressão arterial, dislalia e ansiedade. Exames de imagens constataram depósitos de material ferromagnético nos globos pálidos, putâmens e cabeça dos núcleos caudados associadas a alteração simétrica do sinal das margens dos núcleos basais, da face posterior das cápsulas internas, mesencéfalo, ponte, bulbo e pedúnculos cerebelares superior e médio. Paciente em uso de trientina há 4 anos, encontra-se estável, porém com déficits motores. Conclusões: a DW é uma enfermidade rara e seu diagnóstico precoce implica um elevado nível de suspeita clínica em todos os pacientes, principalmente em jovens que apresentem anormalidades hepáticas e sintomas neuropsiquiátricos atípicos. O diagnóstico precoce é difícil, porém imprescindível para o tratamento específico, melhorando o prognóstico dos pacientes e evitando a progressão da doença para formas graves de doença hepática e neurológicas.
\end{abstract}

Palavras-chave: Doença de Wilson. Metabolismo de cobre. Ceruloplasmina. Gene ATP7B.

ABSTRACT
Introduction: Hepatolenticular degeneration, known as Wilson's disease (WD), is a
rare recessive autosomal disorder of copper metabolism, with a prevalence of 1:30,000
live births, mainly affecting young adults. Patients may present psychomotor,
ophthalmologic disorders, atypical psychiatric diseases, unexplained haemolysis, liver
disease without any other apparent cause. Case report and discussion: A patient
are 27-year-old, male, diagnosed with WD at 22 years of age. He started the condition
complaining of elevation of blood pressure, dyslalia and anxiety. Imaging exams found
deposits of ferromagnetic material in the pale globes, pits and head of the caudata
nuclei associated with symmetrical alteration of the signal from the margins of the basal
nuclei, from the posterior face of the internal capsules, midbrain, bridge, and upper and
middle cerebellar peduncles. Patient is using Trientin for 4 years, is stable, but with
motor deficits. Conclusions: WD is a rare disease and its early diagnosis implies a
high level of clinical suspicion in all patients, especially in young people who present 
hepatic abnormalities and atypical neuropsychiatric symptoms. Early diagnosis is difficult, but indispensable for specific treatment, improving the prognosis of patients and avoiding the progression of the disease to severe forms of liver and neurological disease.

Keywords: Wilson's disease. Copper metabolism disorder. Ceruloplasmin. ATP7B gene

\section{INTRODUÇÃO}

A Degeneração Hepatolenticular, conhecida como Doença de Wilson (DW), é um distúrbio autossômico recessivo raro do metabolismo de cobre (KALER, 2011), que acomete principalmente jovens adultos. Este trabalho tem a intenção de relatar um caso de DW, uma doença rara com prevalência de 1:30.000 nascidos-vivos e realçar a importância de um diagnóstico precoce. A patologia deve entrar como uma das hipóteses diagnósticas em pacientes jovens com distúrbios psicomotores, doenças psiquiátricas atípicas e naqueles com hemólise inexplicada ou com manifestações de doença hepática sem outra causa aparente. Ressaltar ainda a importância de um conhecimento amplo sindrômico e correlacionar tais sintomas com possíveis hipóteses diagnósticos, incluindo a DW, visto que é uma patologia mundialmente distribuída, ademais seu diagnóstico precoce resulta em melhor resultado terapêutico e prognóstico, evitando assim que o paciente desenvolva as várias complicações desencadeadas pelo acúmulo de cobre no organismo, principalmente a Insuficiência Hepática Aguda. A grande dificuldade no diagnóstico precoce consiste na grande diversidade de manifestações clínicas apresentadas pelos pacientes com DW, cujo diagnóstico depende de um alto grau de suspeita clínica com base nos sinais clínicos, achados laboratoriais, presença de anéis de Kayser-Fleisher e histórico familiar (FERNANDES, 2016). O tratamento consiste em medicamentos quelantes de cobre ou que diminuem a absorção intestinal do metal, além de evitar alimentos ricos em cobre e, em últimos casos, pode ser realizado o transplante hepático. O diagnóstico precoce e encaminhamento ágil e adequado para o atendimento especializado dão à Atenção Básica uma propriedade essencial para um melhor prognóstico e resultado terapêutico (MINISTÉRIO DA SAÚDE, 2018). O relato de caso constituiu-se em uma pesquisa de finalidade básica, aplicada, qualitativa e descritiva realizada de maneira longitudinal. Foi realizada através da análise da evolução do quadro clínico de um paciente portador da Doença de Wilson no Centro de Saúde de Catanduvas/PR. A pesquisa documental teve por base o prontuário clínico e exames complementares realizados pelo paciente. A pesquisa bibliográfica teve como base científica a revisão de literatura e artigos científicos encontrados em banco de dados do PUBMED, MEDLINE, SCIELO, entre outros dos últimos 10 anos. Foi encaminhado e aprovado pelo Comitê de Ética com protocolo CAAE: 02730718.20000.5219.

\section{RELATO E DISCUSSÃO DO CASO}

Paciente de 27 anos, sexo masculino, diagnosticado com DW aos 22 anos de idade. Iniciou o quadro com queixa de elevação de pressão arterial, cefaleia, dislalia, ansiedade e diminuição da acuidade visual. Encaminhado ao oftalmologista e neurologista, que solicitou exames de imagens nos quais foram constatados depósito de material ferromagnético nos globos pálidos, putâmens e cabeça dos núcleos caudados associadas a alteração simétrica do sinal das margens dos núcleos basais, da face posterior das cápsulas internas, mesencéfalo, ponte, bulbo e pedúnculos 
cerebelares superior e médio. Exames laboratoriais apresentaram ceruloplasmina e cobre sérico total diminuídos, além do cobre urinário em 24 horas elevado, confirmando o diagnóstico da doença de Wilson. Iniciado tratamento com quelante de cobre, penicilamina, em outubro de 2013, porém houve piora do quadro, sendo suspenso e prescrito, em janeiro de 2015, outro quelante em seu lugar, a trientina na dose de $1000 \mathrm{mg} / \mathrm{dia}$. No início de 2016, paciente iniciou com quadros recorrentes de vômitos biliosos, dor abdominal e vômitos escuros, segundo relatos da mãe, realizado biópsia hepática, sem resultados descritos no prontuário. Em 2018, absteve-se das consultas neurológicas e dos medicamentos. Paciente encontra-se estável, porém com déficits motores. Está em acompanhamento multiprofissional com a equipe de saúde e especialista.

A Degeneração Hepatolenticular, conhecida como Doença de Wilson (DW) (KALER, 2011) foi descrita pela primeira vez por S.A. Kinnear Wilson, em 1912. No Código Internacional de Doenças (CID-10) é classificada como Distúrbio do metabolismo do cobre - E83.0 (MINISTÉRIO DA SAÚDE, 2018). É um distúrbio autossômico recessivo do metabolismo de cobre, que leva ao seu acúmulo basicamente no fígado e no cérebro. A incidência da doença de Wilson é de aproximadamente 1 em 30.000 a 40.000 nascimentos (KALER, 2011; BREWER, 2013), sendo que em populações de casamentos consanguíneos o risco de apresentar traços autossômicos recessivos para a patologia em questão é maior (KALER, 2011).

A maioria dos pacientes apresenta os sintomas na primeira e segunda década de vida, com 5\% desenvolvendo insuficiência hepática aguda (SCHILSKY, 2014), entretanto, em países ocidentais, a apresentação da DW pode se estender até a quinta década de vida (BREWER, 2013).

O principal componente fisiopatológico da DW é a sobrecarga de cobre no fígado, resultante do defeito na excreção biliar do metal (KALER, 2011), bem como seus efeitos tóxicos por lesão oxidativa em outros tecidos (BREWER, 2013). A DW é desencadeada por mutações no gene ATP7B, situado no braço longo do cromossomo 13 (MINISTÉRIO DA SAÚDE, 2018). Esse gene é responsável por codificar a enzima ATPase transportadora de cobre, presente principalmente no fígado, que, por conseguinte, é responsável pelo transporte de cobre através das membranas das organelas intracelulares (MINISTÉRIO DA SAÚDE, 2018). Sendo assim, as mutações nesse gene impedem o transporte de cobre e sua excreção para as vias biliares (KALER, 2011), levando ao seu acúmulo no organismo.

Além do cobre, esse processo envolve uma enzima alfa 2-glicoproteína multifuncional (KALER, 2011), denominada de ceruloplasmina, que é sintetizada no fígado e necessita de 6 átomos de cobre por molécula para se manter estável. $\mathrm{O}$ defeito na incorporação de cobre à cerulopasmina acarreta um excesso de catabolismo e baixos níveis sanguíneos dessa glicoproteína. Geralmente os níveis séricos de cobre são inferiores à faixa normal devido aos baixos níveis plasmáticos de ceruloplasmina, que normalmente liga mais de $90 \%$ do cobre sérico (BREWER, 2013).

Com a progressão da doença, os níveis de cobre livre aumentam, resultando em acúmulo de cobre em outros tecidos (BREWER, 2013). Quando o fígado não suporta mais o acúmulo excessivo de cobre ou quando há dano hepatocelular, o metal é liberado para a circulação sistêmica depositando-se em tecidos extra-hepáticos, principalmente no cérebro (MINISTÉRIO DA SAÚDE, 2018).

O acúmulo de cobre nesses diferentes tecidos é o responsável pela diversidade de manifestações clínicas que os pacientes apresentam como as manifestações 
hepáticas, neurológicas, psiquiátricas, renais, oftalmológicas e outros (MINISTÉRIO DA SAÚDE, 2018).

As manifestações hepáticas podem ser desde assintomáticos até descompensação hepática e hepatite fuminante (MINISTÉRIO DA SAÚDE, 2018; BREWER, 2013). Episódios de hepatite com elevação dos níveis sanguíneos de aminotranferases podem ocorrer, com ou sem icterícia, seguida de remissão espontânea, mas frequentemente verifica-se recidivas de hepatite e a maioria desses pacientes exibirá cirrose mais tarde (BREWER, 2013).

Aproximadamente $50 \%$ dos pacientes com doença neurológica apresentam distúrbios de personalidade, depressão, ansiedade, hiperatividade entre outros (KALER, 2011; BREWER, 2013). Os pacientes com manifestações neurológicas frequentemente exibem distúrbios do movimento como distonia, incoordenação e tremor, quadro clínico que se assemelha muito ao Parkinsonismo (KALER, 2011; MINISTÉRIO DA SAÚDE, 2018; BREWER, 2013).

Cerca de $95 \%$ dos pacientes com sinais neurológicos apresentam córnea com os anéis de Kayser-Fleisher identificados pelo oftalmologista através da lâmpada de fenda (KALER, 2011; BREWER, 2013). Outras manifestações podem ocorrer como: abortos espontâneos, colelitíase e nefrolitíase, danos renais, hemólise, hipoparatiroidismo, miocardiopatias dentre outros (MINISTÉRIO DA SAÚDE, 2018; BREWER, 2013).

O diagnóstico padrão-ouro é a biópsia hepática com ensaio quantitativo de cobre hepático (BREWER, 2013) superiores a $200 \mu \mathrm{g} / \mathrm{g}$ de peso seco do fígado (KALER, 2011; BREWER, 2013). Entretanto, tanto os achados clínicos quanto os laboratoriais são valiosos para o diagnóstico (KALER, 2011; MINISTÉRIO DA SAÚDE, 2018). A presença de anéis de Kayser-Fleisher (Fig.1) são indicativos da doença (KALER, 2011; MINISTÉRIO DA SAÚDE, 2018; BREWER, 2013), contudo, apenas $30 \%$ a $50 \%$ dos pacientes diagnosticados com DW nos estágios hepáticos ou pré-sintomáticos exibem tal achado, ou seja, sua ausência não exclui o diagnóstico (BREWER, 2013).

Figura 1. Anel de Kayser-Fleisher em paciente recém diagnosticado como portador de doença de Wilson (KALLER, 2011).

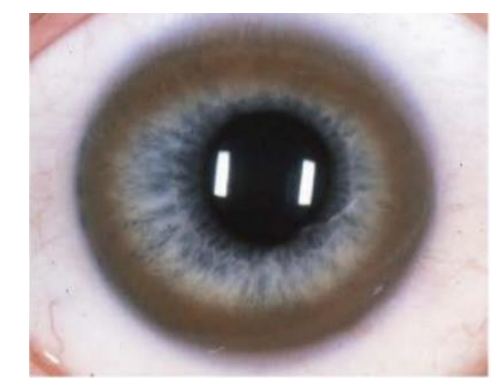

Os achados laboratoriais que auxiliam no diagnóstico incluem baixos níveis séricos de cerulopasmina (KALER, 2011; MINISTÉRIO DA SAÚDE, 2018; BREWER, 2013; SILVÉRIO et al, 2018) - abaixo de $90 \%$ do valor normal de 18 a 35mg/dL - e de cobre (BREWER, 2013), além de níveis elevados de aminotransferases hepáticas, aminoacidúria e anemia hemolítica (KALER, 2011; BREWER, 2013). Além desses, a dosagem do cobre urinário também é importante para o diagnóstico, sendo de fácil realização. Os pacientes com DW apresentam excreção de cobre urinário maior que

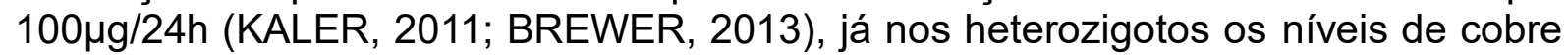
urinário são menores que 80 $\mu \mathrm{g} / 24 \mathrm{~h}$ (BREWER, 2013). 
O teste genético da mutação do gene ATP7B é altamente confiável nas famílias em que os alelos mutantes foram determinados (KALER, 2011), porém a grande variedade de mutações que existem contribuem para falso-positivos (SILVESTRE et al, 2018).

O diagnóstico de imagem com RM revela dilatação ventricular e atrofia difusa do córtex, tronco encefálico e cerebelo. Pacientes com sintomas neurológicos iniciais provavelmente demonstram gânglios da base anormais, principalmente o putâmen, o tálamo, a cabeça do caudado e o globo pálido. Já os paciente com sintomas hepáticos apresentam em imagens T1 ponderadas, um sinal mais forte nos gânglios da base, como também anormalidades no tegumento mesencefálico, na ponte e na substância negra (MENKES, 2011).

Todos os portadores DW diagnosticados, independentemente de serem assintomáticos ou não, necessitam de tratamento (MENKES, 2011). O tratamento consiste primeiramente em remover o excesso de cobre do organismo com quelantes e, secundariamente, em prevenir o reacúmulo tecidual do metal (MINISTÉRIO DA SAÚDE, 2018; MENKES, 2011).

O tratamento inicial para DW deu-se com a penicilamina, um quelante de cobre, que estimula a excreção urinária do metal. Deve ser sempre administrada concomitantemente com vitamina B6 (piridoxina) (KALER, 2011; MINISTÉRIO DA SAÚDE, 2018; BREWER, 2013). Alguns pacientes podem apresentar intolerância a penicilamina devido aos seus efeitos colaterais como nefrotoxicidade e anormalidades hematológicas e, nesses casos, é utilizado a trientina, um quelante alternativo, cujos efeitos colaterais são menores (KALER, 2011; BREWER, 2013).

O acetato de zinco é um medicamento que diminui a absorção intestinal do cobre através do aumento da produção de metalotioneína, proteína produzida no fígado que absorve o cobre do tubo digestivo, impedindo sua absorção pelo organismo e eliminando o metal pelas fezes (KALER, 2011; MINISTÉRIO DA SAÚDE, 2018). Tem grande valor no tratamento de pacientes jovens pré-sintomáticos, em gestantes e como terapia de manutenção (KALER, 2011).

O fármaco de escolha para pacientes com distúrbios neurológicos após tratamento com penicilamina é o tetratiomolibdato que, além de reduzir rapidamente o cobre livre circulante, preservando dessa forma a função neurológica, possui baixa toxicidade (KALER, 2011; BREWER, 2013; FERNANDES, 2016). Seu mecanismo de ação consiste em formar um complexo entre a droga, o cobre e as proteínas da dieta, não permitindo a absorção do metal; além disso, se administrado longe das refeições, atua interagindo com o cobre plasmático (FERNANDES, 2016).

Além do tratamento farmacológico, os pacientes devem ser orientados a manter uma dieta com baixa concentração de cobre (KALER, 2011; MINISTÉRIO DA SAÚDE, 2018; SILVESTRE et al, 2018). Alimentos como marisco, fígado (KALER, 2011; SILVESTRE et al, 2018), chocolate, nozes e cogumelos devem ser evitados (KALER, 2011).

O transplante hepático é uma opção de tratamento (KALER, 2011; MINISTÉRIO DA SAÚDE, 2018; SILVESTRE et al, 2018) reservado apenas em diagnóstico tardio ou baixa adesão ao tratamento farmacológico resultando em dano hepático irreversível (KALER, 2011). Os pacientes com Doença Hepatolenticular correspondem de $6 \%$ a $12 \%$ de todos os pacientes com insuficiência hepática aguda que são encaminhados para transplante hepático de emergência (SILVESTRE et al, 2018).

O paciente com DW deve ter um tratamento contínuo e ininterrupto (KALER, 2011; BREWER, 2013) o que leva a uma recuperação da função hepática depois de um ano de tratamento, embora lesões hepáticas residuais geralmente estejam 
presentes. Os sintomas psiquiátricos e neurológicos geralmente melhoram depois de 6 a 24 meses de tratamento (BREWER, 2013) e os exames de imagem mostram regressão progressiva das áreas anormais dos glânglios da base (BREWER, 2013; MENKES, 2011).

O diagnóstico precoce e encaminhamento ágil e adequado para o atendimento especializado dão à Atenção Básica uma propriedade essencial para um melhor prognóstico e resultado terapêutico (MINISTÉRIO DA SAÚDE, 2018).

\section{CONCLUSÕES}

A Doença de Wilson é uma enfermidade rara e seu diagnóstico precoce implica um elevado nível de suspeita clínica em todos os pacientes que apresentem anormalidades hepáticas e sintomas neuropsiquiátricos atípicos, principalmente se forem jovens. O diagnóstico precoce é difícil, porém imprescindível para o tratamento específico, melhorando o prognóstico dos pacientes e evitando a progressão da doença para formas graves de doença hepática e neurológicas.

\section{REFERÊNCIAS}

BRASIL. Ministério da Saúde. Protocolo clínico e diretrizes terapêuticas: Doença de Wilson. Portaria SAS/MS no 9, de 27 de março de 2018. Disponível em: http://portalarquivos2.saude.gov.br/images/pdf/2018/abril/09/Portaria-ConjuntaPCDT-Doenca-de-Wilson.pdf. Acesso em: 5 set. 2018.

BREWER, G.J. Doença de Wilson. In: Harrison TR (Org.). Medicina Interna de Harrison. 18ed. Porto Alegre: AMGH, 2013.

FAG. Manual para elaboração e apresentação de trabalhos acadêmicos. 4. ed. Cascavel: FAG - Faculdade Assis Gurgacz, 2011.

FERNANDES, V.F.F. Dificuldades do diagnóstico e tratamento da Doença de Wilson. Porto, 2016.38f. Dissertação (Mestrado Integrado em Medicina - Instituto de Ciências Biomédicas Abel Salazar) - Universidade do Porto. Disponível em: https://repositorio-aberto.up.pt/bitstream/10216/90749/2/173684.pdf. Acesso em: 15 mai.2019.

KALER, S.G. Doença de Wilson. In: Goldman L.; Ausiello D.(Org.). Cecil Medicina. 23 ed. Rio de Janeiro: Elsevier, 2011.

MENKES, J.H. Transtornos do metabolismo dos metais: degeneração hepatolenticular. In: Rowland L.P.; Pedley T.A. Merrit tratado de neurologia. 12ed. Rio de Janeiro: Guanabara Koogan, 2011.

SCHILSKY, M. L. Wilson disease: clinical manifestations, diagnosis and treatment. Revista Clinical Liver Disease, vol 3, ed. 5, p. 104-108. Maio, 2104. Disponível em: https://aasldpubs.onlinelibrary.wiley.com/doi/full/10.1002/cld.349. Acesso em: 17 ago. 2018. https://doi.org/10.1002/cld.349

SILVÉRIO, A.S.D.; COUTO, T.S.; OLIVIERA, J.M.P. Síndrome de Wilson: relato de caso. Periódicos Universidade de São Paulo, vol 51, n. 1, p. 75-81. Set, 2018. 
Disponível em: http://www.revistas.usp.br/rmrp/article/view/150085. Acesso em: 21 out. 2018. DOI: https://doi.org/10.11606/issn.2176-7262.v51i1p75-81

SILVESTRE, R.C.; LIMA, M.S.; RIGONI, A.M.; BASTIANELLO, A.P.P.; OLIOSO, A.C.; DANTAS, J.M.M. Doença de Wilson em um adulto jovem: relato de caso e revisão de literatura. Revista Gastroenterologia Endoscopia Digestiva, vol 37, n. 2, p. 21-25. Abr/Jun, 2018. Disponível em: http://sbhepatologia.org.br/pdf/37-01.pdf. Acesso em: 05 set. 2018. 